\title{
Anti-hyperlipidemic effects of the compound Danshen tablet: roles of antioxidation, anti-inflammation, anticoagulation, and anti-apoptosis
}

\author{
Haibiao Guo ${ }^{1,2 \#}$, Lin Chen ${ }^{1 \#}$, Chuyuan $\mathrm{Li}^{2}$, Deqin Wang ${ }^{2}$, Yun Luo ${ }^{1}$, Guibo Sun ${ }^{1}$, Xiaobo Sun ${ }^{1}$ \\ ${ }^{1}$ Institute of Medicinal Plant Development, Peking Union Medical College and Chinese Academy of Medical Sciences, Beijing, China; ${ }^{2}$ Hutchison \\ Whampoa Guangzhou Baiyunshan Chinese Medicine Co., Ltd., Guangzhou, China \\ Contributions: (I) Conception and design: Y Luo, X Sun; (II) Administrative support: G Sun; (III) Provision of study materials or patients: H Guo, \\ C Li; (IV) Collection and assembly of data: L Chen, Y Luo; (V) Data analysis and interpretation: H Guo, D Wang; (VI) Manuscript writing: All \\ authors; (VII) Final approval of manuscript: All authors. \\ \#These authors contributed equally to this work. \\ Correspondence to: Yun Luo; Guibo Sun; Xiaobo Sun. Institute of Medicinal Plant Development, Chinese Academy of Medical Sciences \& Peking \\ Union Medical College, No. 151, Malianwa North Road, Haidian District, Beijing 100193, China. Email: ly20040423@126.com; \\ sunguibo@126.com; sun_xiaobo163@163.com.
}

Background: Hyperlipidemia could cause some serious harm to human health diseases, such as atherosclerosis, coronary heart disease. This study sought to investigate the effects of the compound Danshen tablet (CDT) on hyperlipidemia induced by a high-fat diet in $\mathrm{ApoE}^{-/}$mice and related antioxidation, anti-inflammation, anticoagulation, and anti-apoptosis mechanisms.

Methods: The control group (Group 1) comprised 15 male C57BL/6N mice, and the other 5 groups (Groups 2-6) comprised 75 male ApoE ${ }^{-/-}$mice. These 75 mice were randomly divided into 1 of the following 5 groups: Group 2, a model group; Groups 3-5, the CDT groups, each of which was administered 375, 750 , or $1,500 \mathrm{mg} / \mathrm{kg}$ of CDT; and Group 6, an atorvastatin group, which was administered $5.2 \mathrm{mg} / \mathrm{kg}$ of atorvastatin. All the mice were fed a high-fat diet for 16 weeks and intragastrically administered with CDT or atorvastatin once a day according to their body weight. After 16 weeks, serum was collected, the aorta was isolated, and blood lipid levels were detected. An enzyme-linked immunosorbent assay was used to detect the serum levels of 4-hydroxynonenal (4-HNE), 8-hydroxy-2'-deoxyguanosine (8-OHdG), intercellular adhesion molecule 1 (ICAM-1), monocyte chemoattractant protein 1 (MCP-1), thromboxane B2 (TXB2), tissue plasminogen activator (tPA), and plasminogen activator inhibitor-1 (PAI-1). The thickness of the aortic wall was measured by ultrasonography. Atherosclerotic plaque and endothelial cell apoptosis in the aortic root were evaluated using oil red O staining and terminal dUTP nick-end labeling (TUNEL) assays, respectively. Results: A comparison of mice in the CDT group and mice in the model group showed that CDT significantly inhibited mice's weight gain. CDT reduced the levels of the inflammatory factor ICAM-1 and the oxidative damage molecule 4-HNE. In the coagulation system, CDT significantly increased tPA levels and reduced TXB2 and PAI-1 levels. Ultrasonography showed that CDT increased the thickness of the aortic wall. The oil red $\mathrm{O}$ staining results revealed that CDT significantly ameliorated lipid accumulation in the aortic valve. TUNEL assays indicated that CDT reduced the number of TUNEL-positive cells in the aortic valve.

Conclusions: CDT has a certain protective effect on hyperlipidemia. The mechanism of CDT may be related to antioxidation, anti-inflammation, anticoagulation, and anti-apoptosis.

Keywords: Compound Danshen tablet (CDT); antioxidation; anti-inflammation; anticoagulation; anti-apoptosis

Submitted Dec 09, 2020. Accepted for publication Mar 24, 2021.

doi: $10.21037 /$ atm-20-7915

View this article at: http://dx.doi.org/10.21037/atm-20-7915

(c) Annals of Translational Medicine. All rights reserved. 


\section{Introduction}

Following changes in lifestyle and living standards, the incidence of hyperlipidemia has increased worldwide. Hyperlipidemia induces the development of arteriosclerosis, coronary heart disease, myocardial infarction, diabetes, and other diseases. In the United States (U.S.), more than 53\% of adults have elevated levels of low-density lipoprotein cholesterol (LDL-C), and only a few adults have well controlled levels (1). Hyperlipidemia is a lipid metabolism disorder characterized by increased levels of triglyceride (TG), total cholesterol (TC), LDL-C, and high-density lipoprotein cholesterol (HDL-C) in the blood (2). This disease has been widely explored. The main pathogenesis of hyperlipidemia is related to the following factors: oxidative stress, inflammatory factors, endoplasmic reticulum stress, and intestinal flora imbalance $(3,4)$. It can be treated clinically with statins and other chemical hypolipidemic drugs, which reduce LDL-C, TC, and other factors, but these compounds also induce many adverse effects in patients after treatment. Thus, effective natural plant drugs need to be developed for the treatment of hyperlipidemia.

Oxidative stress, inflammatory factors triggered by changes in blood components, endothelial cell damage, and lipid metabolic disorders are considered key factors leading to hyperlipidemia. In the serum of hyperlipidemic rats, the activities of lipoprotein lipase, hepatic enzyme, superoxide dismutase, glutathione peroxidase, and nitric oxide synthase are low, and the ability to scavenge free radicals is weak, which results in the accumulation of reactive oxygen species (ROS) in the body. ROS accumulation causes oxidative damage, which increases the lipid peroxidation product malondialdehyde (MDA) levels, which in turn results in oxidative stress. Oxidative stress is involved in the occurrence and development of hyperlipidemia (5). The serum expression levels of inflammatory factors, such as interleukin (IL)-1 $\beta$, IL-6, and tumor necrosis factor- $\alpha$ (TNF- $\alpha$ ), C-reactive protein, and monocyte chemoattractant protein-1 (MCP-1) also lead to lipid metabolism disorders and a decreased vascular endothelial function index (6).

In hyperlipidemia, the body's ROS content increases because of an increase in lipid levels in the blood that produces an imbalance between oxidation and antioxidation. Excessive ROS can reduce antioxidant enzymes' activity, promote the oxidation of LDL-C, and lead to the excessive deposition of lipids in the vascular endothelium. The excessive deposition of lipids in blood vessels causes vascular endothelial cell damage, increases the blood aggregation of red blood cells and platelets, increases blood viscosity, reduces blood flow, and facilitates blood lipids' attachment to the vascular wall. Consequently, the local microcirculation disturbance caused by this deposition increases coagulation factors' activity and promotes platelet aggregation, forming a vicious cycle of coagulation (7). Moreover, hyperlipidemia promotes apoptosis by regulating the expression levels of Bcl-2, Bax, caspase- 3 , and TNF- $\alpha$ and increases the deposition of lipids and oxidation products (8), which leads to more complications. Thus, new and effective drugs for hyperlipidemia need to be developed.

Compound Danshen Tablet (CDT), a widely used Chinese herbal compound prescription, was approved by the Chinese Pharmacopoeia in 1990. The main components of CDT are water-soluble tanshinone, Panax notoginseng saponins, and borneol. This compound prescription has been shown to reduce lipids, protect vascular endothelial cells, promote blood circulation, improve blood flow, inhibit myocardial cell apoptosis, and enhance cardiac function $(9,10)$. CDT also contains Salvia miltiorrbiza and notoginsenoside, which have extensive regulatory functions in anti-inflammatory, antioxidant, antitumor, and anticoagulant activities (11). In Panx. notoginseng (Burk.) F. H. Chen, rich triterpenoids elicit a significant anti-inflammatory effect by reducing the levels of inflammatory factors, such as inducible nitric oxide synthase (iNOS), cyclooxygenase-2 (COX-2), IL-1 $\beta$, IL-6, and TNF- $\alpha$ (12). Peng et al. (13) found that Panax notoginsenosides can inhibit the damage to organs and tissues caused by an immune overreaction by inhibiting the expression of inflammatory factors and functioning as an immunomodulatory agent. Other studies have shown that Danshen plays an antioxidant role by scavenging oxygen free radicals (14).

A new metabolite of Danshen Dripping Pills has been found to prevent cardiac fibrosis by inhibiting the NADPH oxidase isoform 2 (NOX2)/ROS/p38 pathway (15). S. miltiorrbiza in CDT can promote apoptosis by blocking the cell cycle and regulating caspase and cytochrome c $(16,17)$. In traditional Chinese medicine (TCM), CDT promotes blood circulation and removes blood stasis. The mechanism of "promoting blood circulation and removing blood stasis" by CDT may involve the inhibition of adhesion molecules' expression. Several studies have shown that tanshinone IIA, the main lipophilic component of CDT, can elicit an anticoagulant effect by enhancing fibrinolytic activities, reducing COX-2 activities, and inhibiting platelet aggregation; total saponins, an active component of $P$. notoginseng, can also inhibit platelet aggregation $(18,19)$. 
Thus, CDT has excellent anticoagulant effects. However, to date, no research appears to have been conducted on the effect of CDT on hyperlipidemia. Thus, in the present study, a hyperlipidemia mouse model was used to observe the antioxidation, anti-inflammatory, anticoagulant, and antiapoptotic functions of CDT in hyperlipidemic mice.

We present the following article in accordance with the ARRIVE reporting checklist (available at http://dx.doi. org/10.21037/atm-20-7915).

\section{Methods}

\section{Reagents and materials}

The following reagents were used in this study: CDT (Hutchison Whampoa Guangzhou Baiyunshan Chinese Medicine Co., Ltd.; Supplementary file); atorvastatin (Pfizer Pharmaceuticals Ltd.); TC, TG, LDL-C, and HDL-C kits (BioSino Bio-Technology \& Science Inc.); 4-hydroxynonenal (4-HNE), 8-hydroxy-2'-deoxyguanosine (8-OHdG), intercellular adhesion molecule 1 (ICAM-1), monocyte chemoattractant protein 1 (MCP-1), thromboxane B2 (TXB2), tissue plasminogen activator (tPA), and plasminogen activator inhibitor-1 (PAI-1) kits (BioLegend, San Diego, CA, USA); TUNEL kit (Roche, Basel, Switzerland), and Triton X-100, oil red O, and sealing agent containing DAPI (Sigma-Aldrich, St. Louis, MO, USA).

\section{Ethical statement}

Experiments were performed under a project license (SLXD-20180411003) granted by animal ethics committee of Institute of Medicinal Plant Development, Chinese Academy of Medical Sciences, in compliance with national guidelines for the care and use of animals.

\section{Animals}

A total of $75 \mathrm{ApoE}^{-/-}$mice and $15 \mathrm{C} 57 \mathrm{BL}$ mice (male, weighing 18-22 g) were fed at the SPF Animal Centre of the Institute of Medicinal Plant Development, Chinese Academy of Medical Sciences. The mice were maintained under special pathogen-free conditions at approximately $22 \pm 1{ }^{\circ} \mathrm{C}$ on a 12 -h light-dark cycle with food and water ad libitum. There were 6 groups: Group 1, the control group, comprised $15 \mathrm{C} 57 \mathrm{BL}$ mice. Seventy-five $\mathrm{ApoE}^{-/-}$mice were randomly divided into one of the following 5 groups:
Group 2, the model group; Groups 3-5, the low-, medium-, and high-dose CDT group, which were administered 375,750 , and $1,500 \mathrm{mg} / \mathrm{kg}$, respectively; and Group 6, the atorvastatin group (which was administered $5.2 \mathrm{mg} / \mathrm{kg}$ of atorvastatin). The mice's body weight in each group was weighed every week, and the treatments were administered by gavage once a day according to body weight. The control and the model groups were given the same dose of normal saline for 16 weeks. All the mice were fed a high-fat diet.

\section{Collection and treatment of samples}

All the mice were administrated for 16 weeks and then fasted for $12 \mathrm{~h}$ on the 16 th week. Blood samples were taken from the eyeballs of the mice and centrifuged at 3,000 rpm. Serum and plasma were stored at $-80{ }^{\circ} \mathrm{C}$, and normal saline was perfused in the heart. The aorta was separated, fixed in $4 \%$ paraformaldehyde, and embedded in the optimal cutting temperature (OCT) compound. A frozen section of the aorta was then made.

\section{Blood lipid level tests}

The serum concentrations of TC, HDL-C, LDL-C, and TG were measured with a Beckman AU480 automatic biochemistry analyzer (California, USA) following the manufacturer's instructions.

\section{Detection of oxidative stress and inflammatory factors}

An enzyme-linked immunosorbent assay (ELISA) was used following the manufacturer's instructions to detect the levels of 4-HNE, 8-OHdG, protein carbon, MCP-1, and ICAM-1.

\section{Measurement of plasma coagulation and fibrinolysis system indicators}

An ELISA was used to detect plasma von Willebrand factor, tissue-type plasminogen activator, plasminogen inhibitor (PAI-1), D-dimer, tissue factor, antithrombin III, and TXB2 following the manufacturer's instructions.

\section{Oil red $O$ staining}

The frozen sections were left at room temperature for $20 \mathrm{~min}$ and then soaked in deionized water for $30 \mathrm{~min}$. The slices were soaked in oil red $\mathrm{O}$ solution and stained at $60^{\circ} \mathrm{C}$ for $45-$ $60 \mathrm{~min}$ (recommended time: $55 \mathrm{~min}$ ). Next, 60\% isopropanol 
was used to differentiate the frozen sections for $40 \mathrm{~s}$. The sections were washed once with ordinary water, and the slices were placed in a hematoxylin and eosin (HE) solution, dyed at room temperature for $20 \mathrm{~s}$, and rinsed with running water. HE was differentiated with hydrochloric acid in ethanol for $10 \mathrm{~s}$, washed with tap water and diluted ammonia water, and returned to blue for $30 \mathrm{~s}$. Glycerin gelatin was placed in boiling water until it dissolved and added dropwise on a specimen slice, which was subsequently sealed with a (large) cover glass and photographed under a microscope.

\section{Determining cardiac dysfunction and measuring the thickness of the aortic arch vessel wall by echocardiography}

The mice were anesthetized with tribromoethanol and fixed on an ultrasound platform after their chest hair was removed. The cardiac function and thickness of the aortic arch (located between the aortic root and the nearest branch of the common carotid artery) were detected by using a $30 \mathrm{MHz}$ scan head interfaced with a Vevo 770 .

\section{TUNEL assay}

In the aortic valve intima, frozen sections of the aortic valve were taken, cleaned with distilled water, and fixed with $4 \%$ paraformaldehyde. Next, $1 \%$ Triton $\mathrm{X}-100$ was added to the TUNEL detection solution for $30 \mathrm{~min}$ and incubated at $37^{\circ} \mathrm{C}$ for $1 \mathrm{~h}$. Subsequently, 4',6-diamidino-2-phenylindole (DAPI) staining was performed, and photographs were taken under a fluorescence microscope.

\section{Statistical analysis}

Data were presented as means \pm standard deviation (SD), and the groups were compared by a one-way analysis of variance (ANOVA) using Tukey's method. A $\mathrm{P}<0.05$ was considered statistically significant. The calculations were performed using GraphPad Prism 6.0.

\section{Results}

\section{Effects of CDT on body weight and blood lipid levels in ApoE ${ }^{-/-}$mice}

Bodyweight and blood lipid levels are basic indices of hyperlipoidemia, and as such, their levels were monitored. As Figure $1 A, B$ show, after continuous intragastric administration for 16 weeks, the mice's weight gain in the model group was significantly lower than that of mice in the CDT and atorvastatin groups. The mice's body weight gain in the middle- and high-dose groups was significantly higher than that of mice in the atorvastatin group. The TC, TG, and LDL-C levels in the model group were significantly higher than those of mice in the control group. Thus, mice in the CDT groups showed significantly reduced TC and LDL-C levels compared to those in the model group (see Figure 1C). These results showed that CDT restored the bodyweight of hyperlipidemic mice and that the effect of CDT was better than that of atorvastatin. Thus, CDT appears to control the blood lipid levels of hyperlipidemic mice by regulating TC and LDL-C levels.

\section{Effects of CDT on oxidative stress and inflammatory factors in $\mathrm{ApoE}^{-/-}$mice}

After continuous intragastric administration for 16 weeks, the levels of 4-HNE, 8-OHdG, ICAM-1, and MCP-1 of mice in the model group were significantly higher than those of mice in the control group. Compared to mice in the model group, mice in the CDT group showed significantly reduced levels of the inflammatory factor ICAM-1 and the oxidative damage molecule 4-HNE (see Figure 2). Thus, CDT appears to regulate the expression of some oxidation and inflammatory factors related to hyperlipidemia.

\section{Effects of CDT on plasma coagulation and fibrinolysis system in ApoE $^{-/-}$mice}

After continuous intragastric administration for 16 weeks, the level of $\mathrm{tPA}$ in the model group was significantly lower than that in the control group, while the levels of TXB2 and PAI-1 in the model group were significantly higher than those in the control group. Compared to mice in the model group, mice in the CDT groups showed a significant increase in tPA level and reduced TXB2 and PAI-1 levels (see Figure 3). Thus, CDT appears to regulate the expression of some coagulation factors associated with hyperlipidemia.

\section{Effects of CDT on cardiac function, aortic wall thickness, and aortic atherosclerotic plaque in $\mathrm{ApoE}^{-/-}$mice}

Hyperlipidemia is accompanied by impaired cardiac function. In this study, cardiac function was first measured by echocardiography. As Figure $4 A, B$ show, after continuous intragastric administration for 16 weeks, CDT significantly ameliorated left ventricular diameter (LVD), ejection 
A

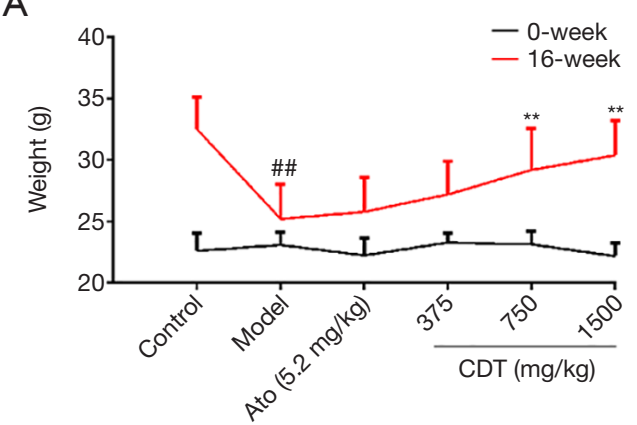

C
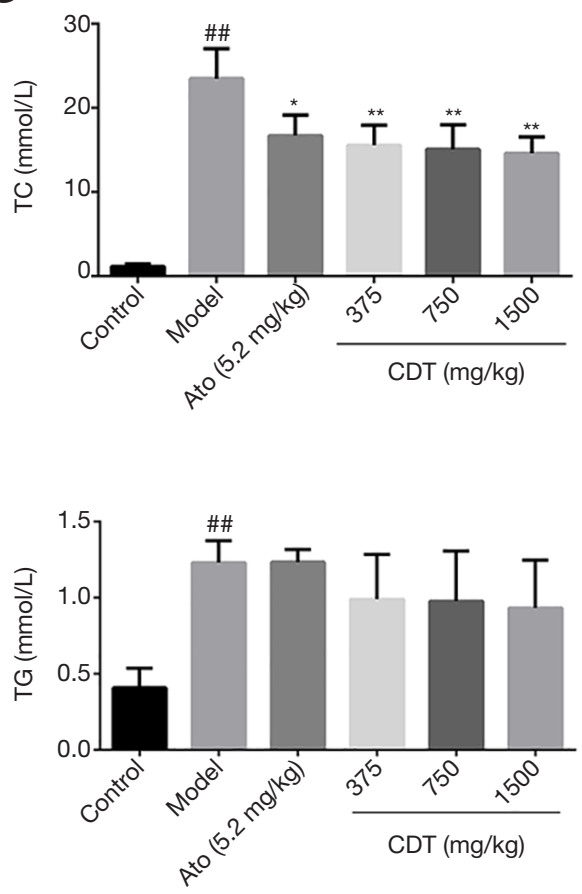

B
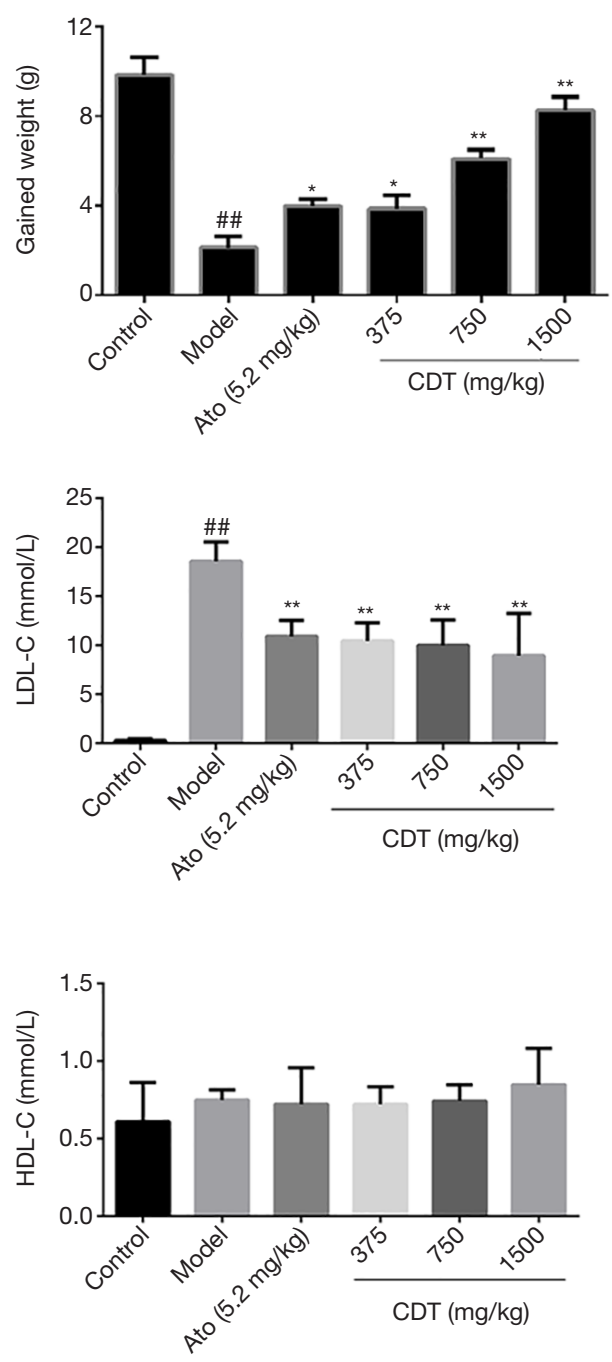

Figure 1 Effects of $\mathrm{CDT}$ on the body weight and blood lipids of $\mathrm{ApoE}^{-/-}$mice. The blood lipid levels were measured with an automatic biochemical analyzer. Data were expressed as means $\pm S D(n=15)$. (A) The weight of each mouse was measured at 0 and 16 weeks. (B) The mice gained weight from the beginning of the study to 16 weeks. (C) The expression levels of TC, LDL-C, TG, and HDL-C in the mice were detected. ${ }^{\#}$. $\mathrm{P}<0.01$, compared with the control group; *, $\mathrm{P}<0.05$, and ${ }^{* *}, \mathrm{P}<0.01$ compared with the model group. CDT, compound Danshen tablet; Ato, atorvastatin; TC, total cholesterol; LDL-C, low-density lipoprotein cholesterol; TG, triglyceride; HDL-C, highdensity lipoprotein cholesterol.

fraction (EF\%), and fractional shortening (FS\%). The thickness of the aortic wall in the model group was significantly higher than that in the control group. The thickness of the aortic wall of mice in the CDT group was significantly reduced compared to that of mice in the model group (see Figure $4 C, D$ ). Thus, CDT appears to improve the cardiac function damage caused by hyperlipidemia, and its effect was shown to be better than that of the positive drug. After continuous intragastric administration for 16 weeks, the number of lipids accumulated in the model group's aortic valve was larger than that of the control group. However, CDT significantly reduced lipid accumulation (see Figure 4E,F).

\section{Effects of CDT on endothelial cell apoptosis in the aortic arch of $A p o E^{-/-}$mice}

Apoptosis is a late event caused by hyperlipidemia. In this 

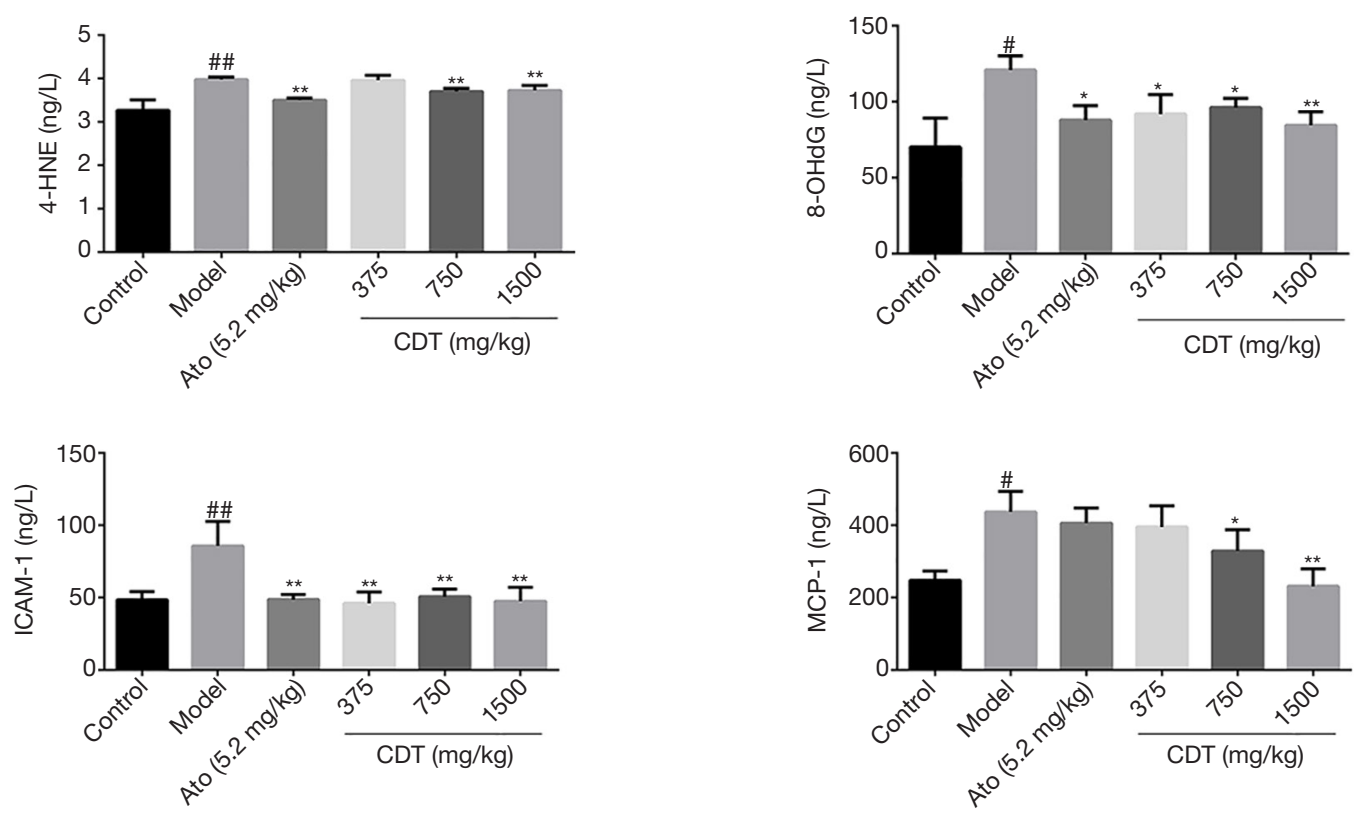

Figure 2 Effects of CDT on 4-HNE, 8-OHdG, ICAM-1, and MCP-1 expression levels at 16 weeks in high-fat diet-fed ApoE ${ }^{-/}$mice. Serum was prepared to determine the levels of 4-HNE, 8-OHdG, ICAM-1, and MCP-1 by an ELISA. Data were expressed as means \pm $\mathrm{SD}(\mathrm{n}=5) .{ }^{*}, \mathrm{P}<0.05$, and ${ }^{\# \#}, \mathrm{P}<0.01$ compared with the control group; * $\mathrm{P}<0.05$, and **, $\mathrm{P}<0.01$ compared with the model group. CDT, compound Danshen tablet; Ato, atorvastatin; 4-HNE, 4-hydroxynonenal; 8-OHdG, 8-hydroxy-2'-deoxyguanosine; ICAM-1, intercellular adhesion molecule 1; MCP-1, monocyte chemoattractant protein-1.
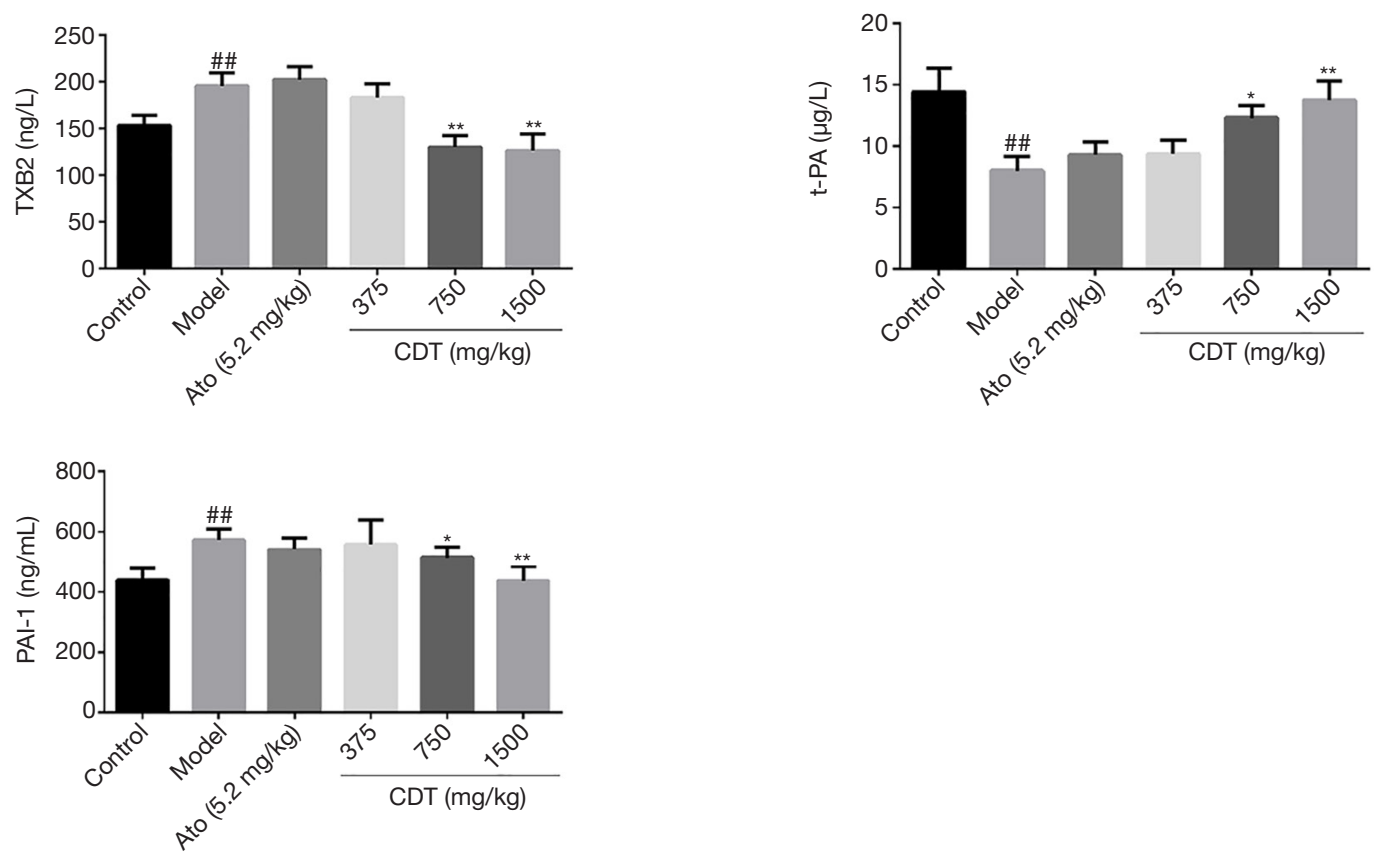

Figure 3 Effects of CDT on TXB2, tPA, and PAI-1 expression levels at 16 weeks in high-fat diet-fed ApoE ${ }^{-/-}$mice. Plasma was prepared to determine the levels of TXB2, tPA, and PAI-1 by an ELISA. Data were expressed as means $\pm \mathrm{SD}(\mathrm{n}=5)$. ${ }^{\#}, \mathrm{P}<0.01$ compared with the control group; * $\mathrm{P}<0.05$, and ** $\mathrm{P}<0.01$ compared with the model group. CDT, compound Danshen tablet; Ato, atorvastatin; TXB2, thromboxane $\mathrm{B} 2$; tPA, tissue plasminogen activator; PAI-1, plasminogen activator inhibitor-1. 
A

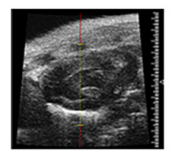

Long axial section of LV

B

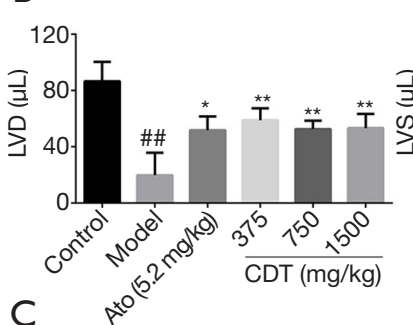

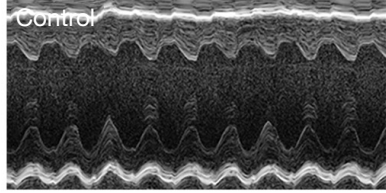

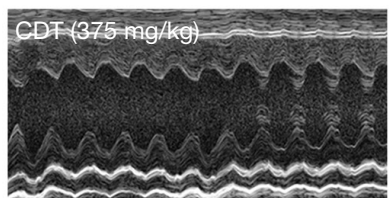

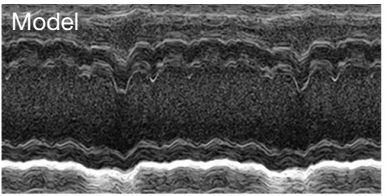

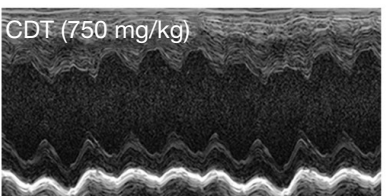

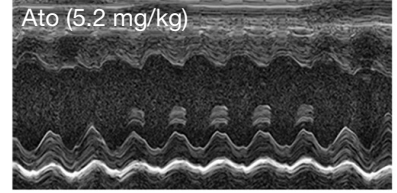

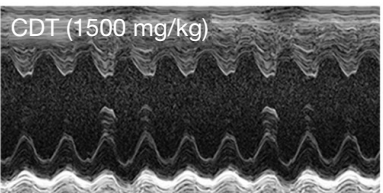

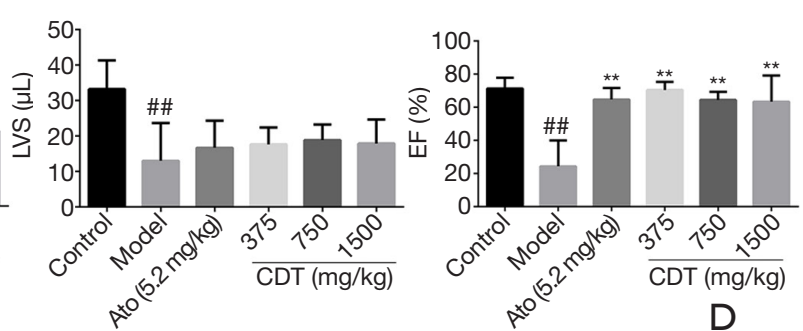
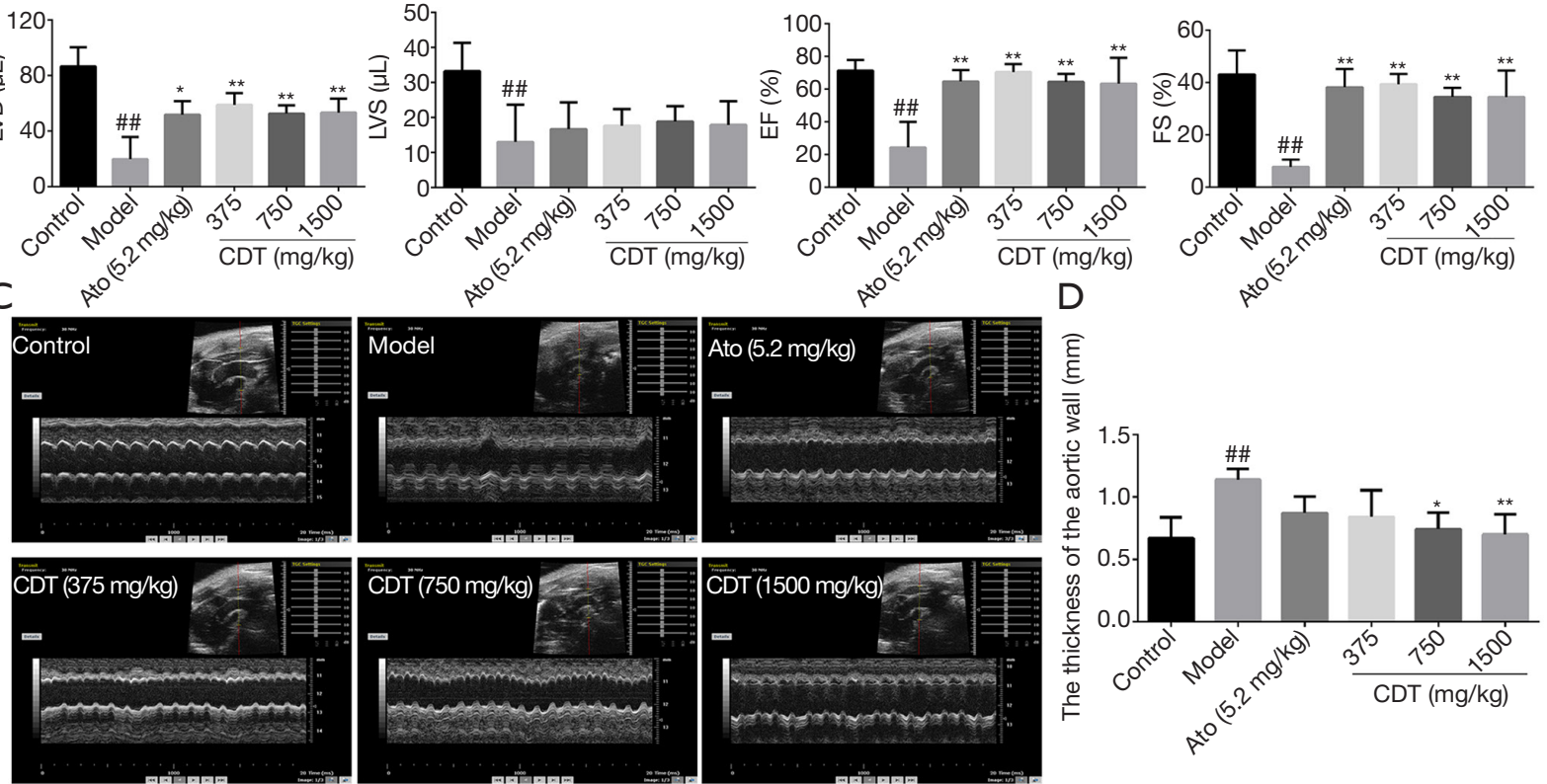

E
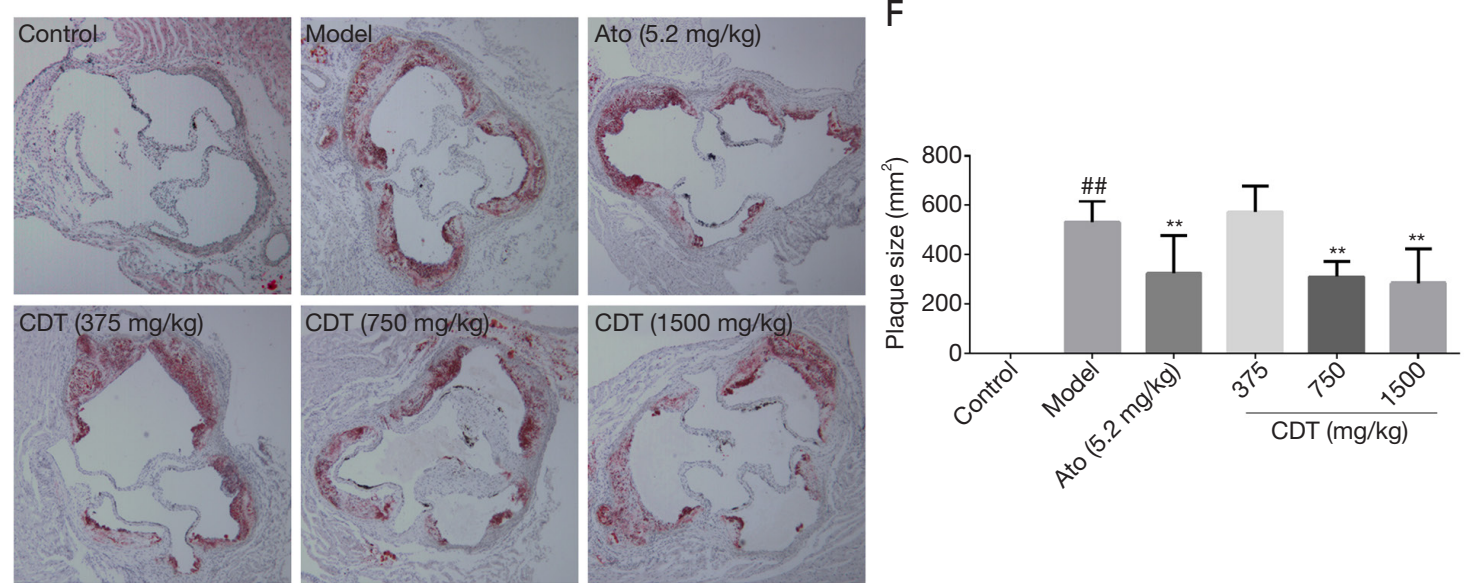

Figure $4 \mathrm{Effects}$ of CDT on the cardiac function, aortic wall thickness, and aortic atherosclerotic plaque of $\mathrm{ApoE}^{-/-}$mice. All the mice were anesthetized with tribromoethanol and evaluated using an ultrasound imaging system. (A) Representative M-mode echocardiography images were shown. (B) LVD, LVS, EF and FS values were calculated. (C) The aortic wall thickness of the mice was detected. (D) The aortic wall thickness was statistically analyzed. (E) The frozen sections of the aortic root were stained with oil red $\mathrm{O}$ (magnification, $\times 40$ ). (F) The aortic wall thickness was statistically evaluated. Data were expressed as means $\pm \mathrm{SD}(\mathrm{n}=6) .{ }^{. \#}, \mathrm{P}<0.01$, compared with the control group; *, $\mathrm{P}<0.05$, and ${ }^{* *}, \mathrm{P}<0.01$ compared with the model group. CDT, compound Danshen tablet; Ato, atorvastatin; LVD, left ventricular diameter; LVS, left ventricular end systolic dimension; EF, ejection fraction; FS, fractional shortening. 

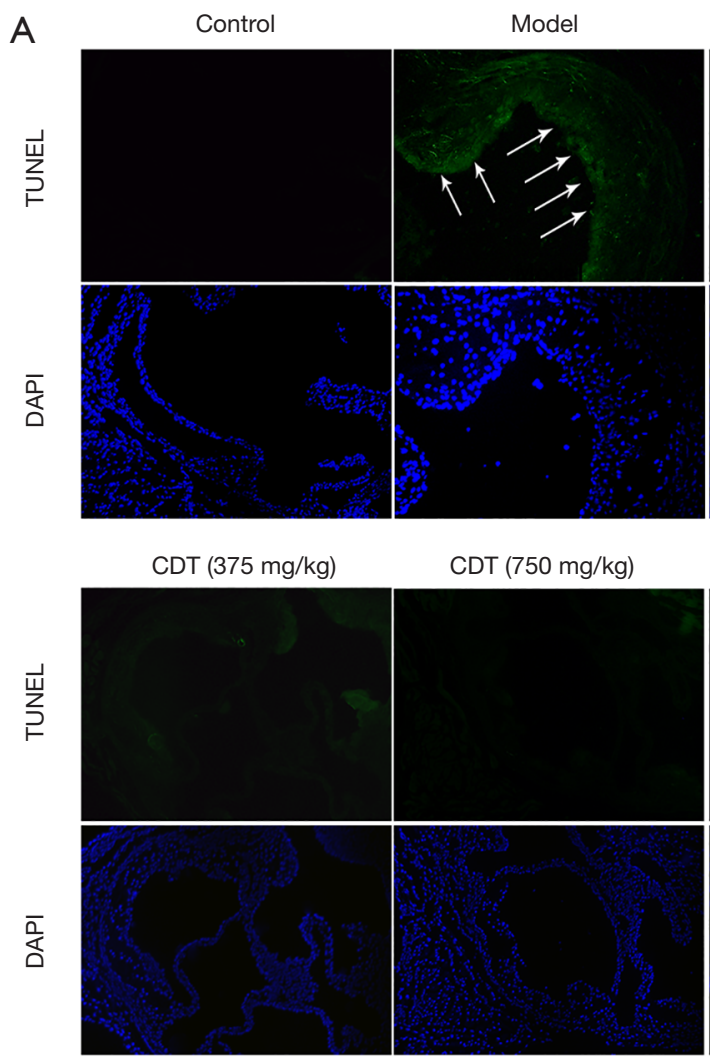

Model

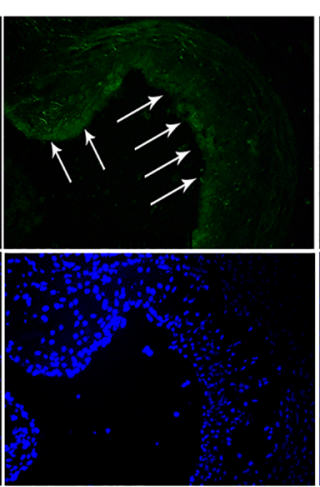

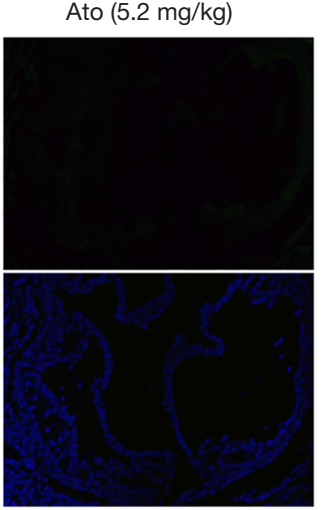

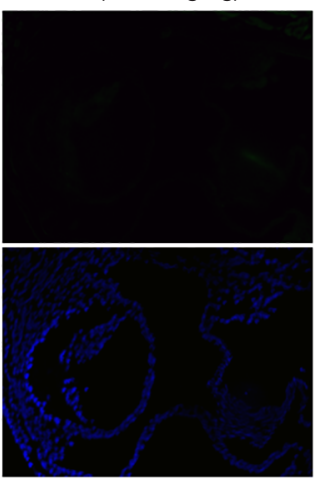

B

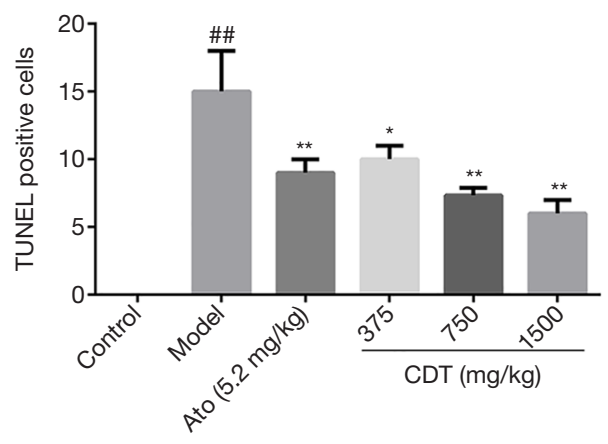

Figure 5 Effects of CDT on endothelial cell apoptosis in the aortic root of high-fat diet-fed ApoE ${ }^{-/-}$mice. The frozen sections of the aortic root were stained by a TUNEL assay. (A) Representative TUNEL images were shown. (B) TUNEL-positive cells were calculated. Data were expressed as means $\pm \mathrm{SD}(\mathrm{n}=6) .^{\#}, \mathrm{P}<0.01$, compared with the control group; * $\mathrm{P}<0.01$, and **, $\mathrm{P}<0.01$ compared with the model group; CDT, compound Danshen tablet; Ato, atorvastatin.

study, after continuous intragastric administration for 16 weeks, the number of TUNEL-positive cells in the model group's aortic valve was significantly higher than that of the control group. Mice in the CDT group showed a significant reduction in the number of apoptotic cells compared to that of mice in the model group, and the number of apoptotic cells in the middle- and high-dose groups were much lower than that in the atorvastatin group (see Figure 5).

\section{Discussion}

Due to changes in lifestyle and eating habits, hyperlipidemia's morbidity has become a serious threat to human health. Hyperlipidemia can induce atherosclerosis, fatty liver, and other diseases. Thus, blood lipid levels need to be controlled safely and effectively. Currently, the first choice of treatment for hyperlipidemia is statins. These drugs effectively reduce blood lipid levels and greatly lower the morbidity of cardiovascular disease. Unfortunately, they also have several side effects, including statin-associated muscle symptoms.

CDT is composed of $S$. miltiorrhiza extract, $P$. notoginseng, and borneol. According to TCM theory, S. miltiorrbiza is a monarch medicine, $P$. notoginseng is a ministerial medicine, and borneol is an adjuvant. CDT possesses various pharmacological effects, such as dilating the coronary artery, protecting the myocardium, improving hemorheology, and exerting antiarrhythmic, hypolipidemic, and liver-protecting effects. It is used to treat cardiovascular, cerebrovascular, and central nervous system diseases $(9,20)$. The active components of CDT can inhibit atherosclerosis formation by reducing lipid deposition in the aorta and decreasing TC and serum lipid levels $(21,22)$. Zhang et al. showed that $P$. notoginseng saponins could regulate the lipid metabolism pathway and inhibit atherosclerosis in rats (23). The active components of $S$. miltiorrbiza can also inhibit the inflammatory reaction and apoptosis of vascular endothelial 


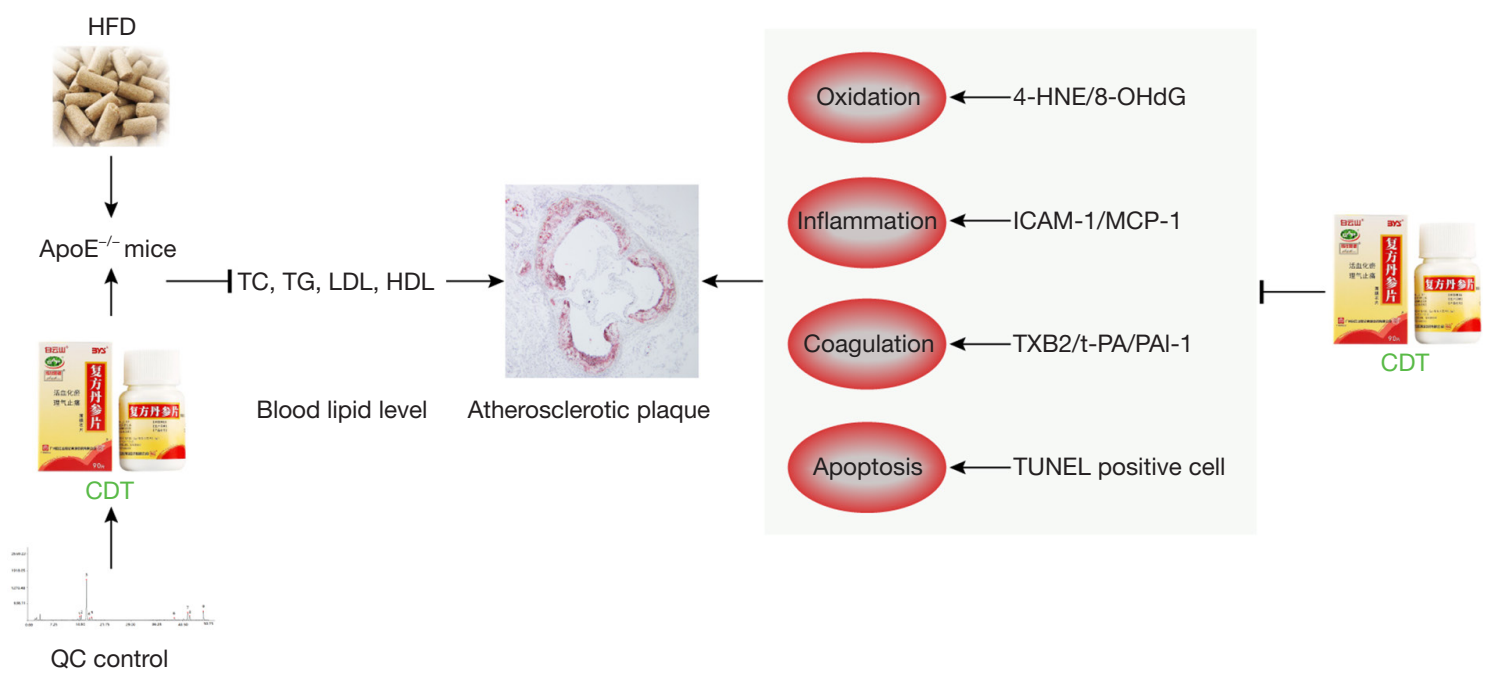

Figure 6 Hypothetical mechanism of CDT against hyperlipemia ApoE ${ }^{-/}$mice. CDT, compound Danshen tablet; 4-HNE, 4-hydroxynonenal; 8-OHdG, 8-hydroxy-2'-deoxyguanosine; ICAM-1, intercellular adhesion molecule 1; MCP-1, monocyte chemoattractant protein-1; TXB2, thromboxane B2; tPA, tissue plasminogen activator; PAI-1, plasminogen activator inhibitor-1.

cells $(24,25)$.

The present study's data showed that the amount of weight gained by mice in the model group decreased significantly after 16 weeks of continuous feeding with a high-fat diet. Conversely, the serum TC and LDL-C levels of mice in the model group increased significantly compared to those of mice in the control group. Thus, CDT significantly improved the changes in the weight of the mice and reduced their serum TC and LDL-C levels. Oxidation stress and inflammation reaction are two key factors associated with hyperlipidemia. The serum ELISA results showed that CDT significantly reduced the expression levels of 4-HNE, 8-OHdG, ICAM-1, and $\mathrm{MCP}-1$. Coagulation factors play an important role in the formation of hyperlipidemia. CDT significantly increased the tPA levels and reduced TXB2 and PAI-1 levels. Thus, CDT exerted its anti-hyperlipidemic effects by reducing the expression of oxidative enzymes and inflammatory factors and regulating coagulation factors.

Mice's cardiac function and aortic wall thickness were further measured by echocardiography. The results showed that CDT reduced the thickness of the aortic wall. Oil red O staining confirmed that CDT significantly reduced the lipid deposition in the mice's aortic valve. A TUNEL assay was conducted to explore the mechanism of CDT against hyperlipidemia, and the results showed that CDT reduced vascular endothelial cell apoptosis in the aortic valve of the mice. Thus, CDT significantly reduced lipid accumulation in the aortic valve, and the underlying mechanisms might be related to the anti-apoptosis effect of CDT on endothelial cells.

In conclusion, CDT improves hyperlipidemia by regulating antioxidation, anti-inflammation, anticoagulation, and anti-apoptosis (see Figure 6). However, the exact molecular targets of CDT need to be clarified based on TCM theory in further studies. Future research should examine endothelial protection and lipid metabolism targets. Generally, this study provides an experimental basis for the clinical use of CDT in the prevention and treatment of hyperlipidemia.

\section{Acknowledgments}

Funding: This work was financially supported by the Research and Development Plan in Key Areas of Guangdong Province (No. 2020B1111110002) and the Major Scientific and Technological Special Project "Drug Innovation Major Project" (No. 2018ZX09711001-009).

\section{Footnote}

Reporting Checklist: The authors have completed the ARRIVE reporting checklist. Available at http://dx.doi. org/10.21037/atm-20-7915

Data Sharing Statement: Available at http://dx.doi. 


\section{Page 10 of 11}

org/10.21037/atm-20-7915

Conflicts of Interest: All authors have completed the ICMJE uniform disclosure form (available at http://dx.doi. org/10.21037/atm-20-7915). The authors have no conflicts of interest to declare.

Ethical Statement: The authors are accountable for all aspects of the work in ensuring that questions related to the accuracy or integrity of any part of the work are appropriately investigated and resolved. Experiments were performed under a project license (SLXD-20180411003) granted by animal ethics committee of Institute of Medicinal Plant Development, Chinese Academy of Medical Sciences, in compliance with national guidelines for the care and use of animals.

Open Access Statement: This is an Open Access article distributed in accordance with the Creative Commons Attribution-NonCommercial-NoDerivs 4.0 International License (CC BY-NC-ND 4.0), which permits the noncommercial replication and distribution of the article with the strict proviso that no changes or edits are made and the original work is properly cited (including links to both the formal publication through the relevant DOI and the license). See: https://creativecommons.org/licenses/by-nc-nd/4.0/.

\section{References}

1. Karr S. Epidemiology and management of hyperlipidemia. Am J Manag Care 2017;23:S139-48.

2. Mogensen SS, Schmiegelow K, Grell K, et al. Hyperlipidemia is a risk factor for osteonecrosis in children and young adults with acute lymphoblastic leukemia. Haematologica 2017;102:e175-8.

3. Bozkurt B, Aguilar D, Deswal A, et al. Contributory Risk and Management of Comorbidities of Hypertension, Obesity, Diabetes Mellitus, Hyperlipidemia, and Metabolic Syndrome in Chronic Heart Failure: A Scientific Statement From the American Heart Association. Circulation 2016;134:e535-78.

4. Panahi $Y$, Ahmadi $Y$, Teymouri $M$, et al. Curcumin as a potential candidate for treating hyperlipidemia: A review of cellular and metabolic mechanisms. J Cell Physiol 2018;233:141-52.

5. Gong G, Qin Y, Huang W, et al. Protective effects of diosgenin in the hyperlipidemic rat model and in human vascular endothelial cells against hydrogen peroxide-

\section{Guo et al. Anti-hyperlipidemic effects of compound Danshen tablet}

induced apoptosis. Chem Biol Interact 2010;184:366-75.

6. Papapanagiotou A, Siasos G, Kassi E, et al. Novel Inflammatory Markers in Hyperlipidemia: Clinical Implications. Curr Med Chem 2015;22:2727-43.

7. Li R, Zhao L, $\mathrm{Wu} \mathrm{N}$, et al. Proteomic analysis allows for identifying targets of Yinchenwuling Powder in hyperlipidemic rats. J Ethnopharmacol 2016;185:60-7.

8. Zhao XS, Wu Q, Peng J, et al. Hyperlipidemia-induced apoptosis of hippocampal neurons in apoE(-/-) mice may be associated with increased PCSK9 expression. Mol Med Rep 2017;15:712-8.

9. Sun Y, Yang J. A bioinformatics investigation into the pharmacological mechanisms of the effect of Fufang Danshen on pain based on methodologies of network pharmacology. Sci Rep 2019;9:5913.

10. Wu P, Zhang Z, Ma G, et al. Transcriptomics and metabolomics reveal the cardioprotective effect of Compound Danshen tablet on isoproterenolinduced myocardial injury in high-fat-diet fed mice. J Ethnopharmacol 2020;246:112210.

11. Xie W, Meng X, Zhai Y, et al. Panax Notoginseng Saponins: A Review of Its Mechanisms of Antidepressant or Anxiolytic Effects and Network Analysis on Phytochemistry and Pharmacology. Molecules 2018;23:940.

12. Jang HJ, Lee S, Lee SJ, et al. Anti-inflammatory Activity of Eudesmane-Type Sesquiterpenoids from Salvia plebeia. J Nat Prod 2017;80:2666-76.

13. Peng XX, Zhang SH, Wang XL, et al. Panax Notoginseng flower saponins (PNFS) inhibit LPS-stimulated NO overproduction and iNOS gene overexpression via the suppression of TLR4-mediated MAPK/NF-kappa B signaling pathways in RAW264.7 macrophages. Chin Med 2015;10:15.

14. Jiang G, Liu J, Ren B, et al. Anti-tumor and chemosensitization effects of Cryptotanshinone extracted from Salvia miltiorrhiza Bge. on ovarian cancer cells in vitro. J Ethnopharmacol 2017;205:33-40.

15. Yin Q, Lu H, Bai Y, et al. A metabolite of Danshen formulae attenuates cardiac fibrosis induced by isoprenaline, via a NOX2/ROS/p38 pathway. Br J Pharmacol 2015;172:5573-85.

16. Wang X, Gao A, Jiao Y, et al. Antitumor effect and molecular mechanism of antioxidant polysaccharides from Salvia miltiorrhiza Bunge in human colorectal carcinoma LoVo cells. Int J Biol Macromol 2018;108:625-34.

17. Cao Y, Huang B, Gao C. Salvia miltiorrhiza extract dihydrotanshinone induces apoptosis and inhibits 
proliferation of glioma cells. Bosn J Basic Med Sci 2017;17:235-40.

18. Lau AJ, Toh DF, Chua TK, et al. Antiplatelet and anticoagulant effects of Panax notoginseng: comparison of raw and steamed Panax notoginseng with Panax ginseng and Panax quinquefolium. J Ethnopharmacol 2009;125:380-6.

19. Yin QS, Chen L, Mi RH, et al. Efficacy and Safety of Danshen Compound Tablets in Preventing ThalidomideAssociated Thromboembolism in Patients with Multiple Myeloma: A Multicenter Retrospective Study. Med Sci Monit 2016;22:3835-42.

20. Teng Y, Zhang MQ, Wang W, et al. Compound danshen tablet ameliorated abeta25-35-induced spatial memory impairment in mice via rescuing imbalance between cytokines and neurotrophins. BMC Complement Altern Med 2014;14:23.

21. Liu X, Guo CY, Ma XJ, et al. Anti-inflammatory effects of tanshinone IIA on atherosclerostic vessels of

Cite this article as: Guo H, Chen L, Li C, Wang D, Luo Y, Sun G, Sun X. Anti-hyperlipidemic effects of the compound Danshen tablet: roles of antioxidation, anti-inflammation, anticoagulation, and anti-apoptosis. Ann Transl Med 2021;9(9):744. doi: 10.21037/atm-20-7915 ovariectomized ApoE mice are mediated by estrogen receptor activation and through the ERK signaling pathway. Cell Physiol Biochem 2015;35:1744-55.

22. Wang J, He X, Chen W, et al. Tanshinone IIA protects mice against atherosclerotic injury by activating the TGF-beta/PI3K/Akt/eNOS pathway. Coron Artery Dis 2020;31:385-92.

23. Zhang YG, Zhang HG, Zhang GY, et al. Panax notoginseng saponins attenuate atherosclerosis in rats by regulating the blood lipid profile and an anti-inflammatory action. Clin Exp Pharmacol Physiol 2008;35:1238-44.

24. Stumpf C, Fan Q, Hintermann C, et al. Anti-inflammatory effects of danshen on human vascular endothelial cells in culture. Am J Chin Med 2013;41:1065-77.

25. Zhou P, Lu S, Luo Y, et al. Attenuation of TNF-alphaInduced Inflammatory Injury in Endothelial Cells by Ginsenoside Rb1 via Inhibiting NF-kappaB, JNK and p38 Signaling Pathways. Front Pharmacol 2017;8:464. 


\section{Supplementary}

\section{HPLC analysis (Figure S1)}

Chromatographic analysis was performed through an Agilent 1260 HPLC system coupled with diode array detector (Agilent Technologies, Palo Alto, CA, USA) according to a previous study (26). Chromatographic data was processed by Agilent Chem Station software. Chromatographic separation was performed on a Diamonsil C18-column $(250 \times 4.6 \mathrm{~mm}, 5 \mu \mathrm{m})$. The mobile phase consisted of acetonitrile (A) and $0.02 \%$ phosphoric acid in water (B), and the flow rate was at $0.6 \mathrm{~mL} / \mathrm{min}$. The eluting conditions were listed as follows: 0-6 min at $61 \% \mathrm{~A} ; 6-20 \mathrm{~min}$ from $61 \%$ to $90 \% \mathrm{~A} ; 20-20.5 \mathrm{~min}$ from $90 \%$ to $61 \%$ A; $20.5-$ $25 \mathrm{~min}$ at $61 \% \mathrm{~A}$. The detection wave length was set at $270 \mathrm{~nm}$.

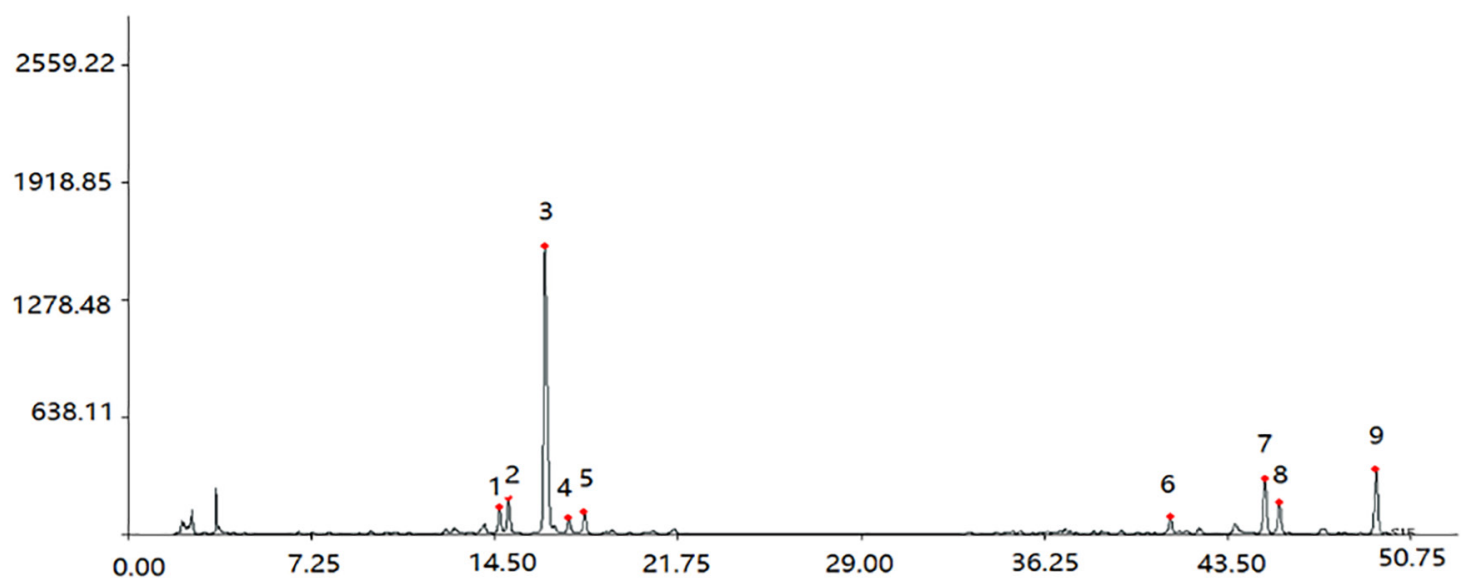

Figure S1 Base peak chromatograms of the main components in CDT. Peak no.: 1. rosmarinic acid; 2. alkannic acid; 3 . salvianolic acid B; 7. Cryptotanshinone; 8. tanshinone I; 9. tanshinone IIA. CDT, compound Danshen tablet.

\section{References}

26. Liu J, Guo Y, Zhang J, et al. Systematic chemical analysis of flavonoids in the Nelumbinis stamen. Phytomedicine 2014;21:1753-8. 